\title{
Potential of mean force and the charge reversal of rodlike polyions
}

\author{
Jürgen F. Stilck \\ Instituto de Física - UFF \\ Av. Litorânea s/n \\ 24.210-340 - Niterói - RJ \\ and Yan Levin \\ Instituto de Física - UFRGS, \\ Caixa Postal 15051, CEP 91501-970, \\ Porto Alegre, Brazil
}

(Dated: December 11, 2018)

\begin{abstract}
A simple model is presented to calculate the potential of mean force between a polyion and a multivalent counterion inside a polyelectrolyte solution. We find that under certain conditions the electrostatic interactions can lead to a strong attraction between the polyions and the multivalent counterions, favoring formation of overcharged polyion-counterion complexes. It is found that small concentrations of salt enhance the overcharging while an excessive amount of salt hinders the charge reversal. The kinetic limitations to overcharging are also examined.
\end{abstract}




\section{INTRODUCTION}

It is our pleasure to contribute this paper to the special issue of Molecular Physics dedicated to celebrate Ben Widom's outstanding contributions to Physical Chemistry and Statistical Mechanics. Ben's work is characterized by a profound physical insight, combined with an ability to abstract the most complex physical phenomena into a simple model. From scaling and criticality ${ }^{\underline{1}}$ to microemulsions ${ }^{2}$ and the hydrophobic effect ${ }^{3}$, Ben's sagacity has opened new frontiers of Physical Chemistry. While it is impossible to compete with Ben's intuition, one can at least try to follow his example. In this paper we will, therefore, study a simple model of interaction between a polyion and multivalent counterions inside a polyelectrolyte solution.

Thermodynamic systems in which long range Coulomb interactions play the dominant role pose an outstanding challenge to Physical Chemistry $\stackrel{4}{*}$. Even such basic question as the possible existence of a liquid-gas phase separation in a restricted primitive model has been positively settled only quite recently $\stackrel{4}{*}$. Even so, the order of this transition still remains a source of an outstanding debate ${ }^{\frac{5}{2}}$. For strongly asymmetric electrolytes such as aqueous colloidal suspensions, even the existence of a liquid-liquid phase separation continues to be controversial $6 \cdot 7 \cdot 8 \cdot 9 \cdot 10.11$.

When aqueous colloidal suspensions or polyelectrolyte solutions contain multivalent counterions other curious phenomena appear. For example, it is found that for sufficiently small separations two like-charged polyions can attract one another ${ }^{12.13 .14 .15 .16 .17 .18 .19}$. If an external electric field is applied to such a suspension the electrophoretic mobility of colloidal particles is often found to be reversed, so that the particles move in the direction opposite to the one expected based purely on their chemical charge 4.20 .21 .22 .23 . Both of these phenomena are a consequence of strong electrostatic coupling between the polyions and the counterions.

The counterions inside the suspension can be divided into two categories: those which are associated (condensed) to the colloidal particle and those which are free. The condensed counterions contribute to the effective, renormalized, charge of the polyion-counterion complex, while the free counterions and coions result in screening of the electrostatic interactions inside the suspension $\stackrel{4}{*}$. In this paper we will explore the potential of mean force between a rodlike polyion with $n$ associated counterions and a counterion located at a transverse distance $d$ from the polyion center, Fig. 1 . 


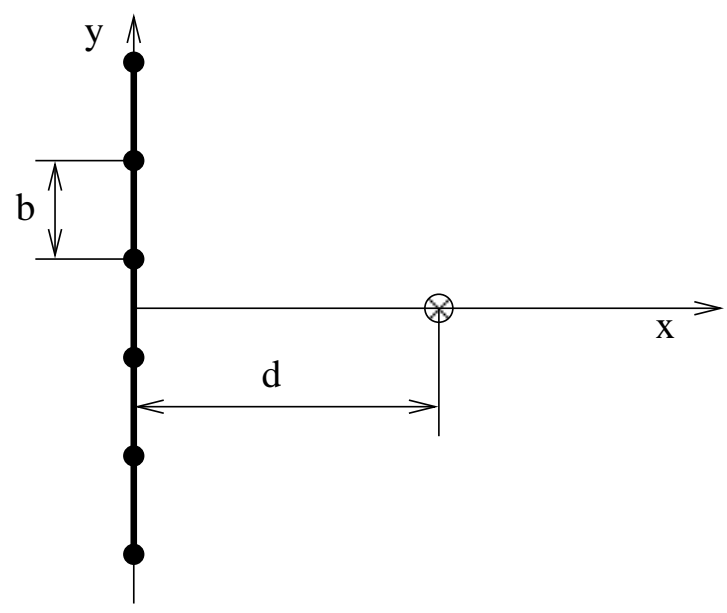

FIG. 1: Rodlike polyion with $Z=6$ monomers separated by a distance $b$ and a counterion located at $\mathrm{x}=\mathrm{d}$.

\section{THE MODEL}

Consider a rodlike polyion of $Z$ (even) monomers, each carrying a charge $-q$, inside an aqueous suspension containing multivalent counterions and salt. The monomers are located uniformly with separation $b$ along the rod. Strong electrostatic coupling between the polyion and the counterions results in a condensation of $n \alpha$-valent counterions onto the polyion. The condensed counterions are free to hop between the monomers of the polyion ${ }^{15}$. If a monomer has an associated counterion, its charge is renormalized to $(\alpha-1) q$. The free, uncondensed, counterions and coions screen the electrostatic interactions, changing the potential between the two charges $q_{1}$ and $q_{2}$ from the Coulomb to the Debye-Hückel24 form

$$
V(r)=\frac{1}{\epsilon} \frac{q_{1} q_{2} \exp (-\kappa r)}{r}
$$

where $\epsilon$ is the dielectric constant of the solvent and $\kappa$ is the inverse Debye length. The question that we would like to address in this paper is what is the potential of mean force between the polyion-counterion complex containing $n$ condensed $\alpha$-ions and an additional $\alpha$-valent counterion located transversely at distance $d$ from the polyion center, see Fig. 1 .

To proceed, we assign to each monomer $i$ a lattice-gas variable $\sigma_{i}$, such that $\sigma_{i}$ is equal to 1 if a counterion is condensed onto cite $i$ and 0 otherwise. For a given configuration $\{\sigma\}$, the interaction Hamiltonian between the complex and a counterion located at a transverse 
distance $d$ from its center is

$$
\begin{aligned}
\mathcal{H}= & \frac{1}{D} \sum_{i=1}^{Z} \frac{\alpha q^{2}\left(\sigma_{i} \alpha-1\right)}{\sqrt{r_{i}^{2}+d^{2}}} \exp \left(-\kappa \sqrt{r_{i}^{2}+d^{2}}\right) \\
& +\frac{1}{2 D} \sum_{i, i^{\prime}=1, i \neq i^{\prime}}^{Z} \frac{q^{2}\left(\sigma_{i} \alpha-1\right)\left(\sigma_{i^{\prime}} \alpha-1\right)}{b\left|i-i^{\prime}\right|} \exp \left(-\kappa b\left|i-i^{\prime}\right|\right),
\end{aligned}
$$

where

$$
r_{i}=\frac{2 i-1-Z}{2} b
$$

It is convenient to define the reduced distance between the polyion and the counterion $x=d / b$, the reduced inverse Debye length $k=\kappa b$, and the Manning parameter ${ }^{25.26}$ as $\xi=$ $q^{2} / \epsilon k_{B} T b$. In terms of these adimensional variables the reduced Hamiltonian, $H \equiv \beta \mathcal{H} / \xi$, becomes

$$
\begin{aligned}
H= & \sum_{i=1}^{Z}\left(\sigma_{i} \alpha-1\right)\left[\frac{2 \alpha}{\sqrt{(2 i-1-Z)^{2}+4 x^{2}}} \exp \left(-k \sqrt{(2 i-1-Z)^{2}+4 x^{2}}\right)\right. \\
& \left.+\frac{1}{2} \sum_{i^{\prime}=1, i \neq i^{\prime}}^{Z} \frac{\sigma_{i^{\prime}} \alpha-1}{\left|i-i^{\prime}\right|} \exp \left(-k\left|i-i^{\prime}\right|\right)\right] .
\end{aligned}
$$

The partition function is a trace over all possible distributions of $n$ condensed counterions among the $Z$ polyion sites. There is a total of

$$
N_{c}=\frac{Z !}{(Z-n) ! n !}
$$

such configurations. The partition function is then

$$
Q=\sum_{\{\sigma\}}^{\prime} \exp [-\xi H]
$$

where the sum is over the $N_{c}$ configurations $\{\sigma\}$ which obey the constraint $\sum_{i=1}^{Z} \sigma_{i}=n$, denoted by the prime. It is convenient to order the terms in the Hamiltonian by the distances between the pair of interacting charges. This results in

$$
\begin{aligned}
H=\sum_{i=1}^{Z / 2} 2 \alpha[( & \left.\left.\sigma_{i}+\sigma_{Z-i+1}\right) \alpha-2\right]\left[\frac{\exp \left(-k \sqrt{\left.(2 i-1-Z)^{2}+4 x^{2}\right)}\right.}{\sqrt{(2 i-1-Z)^{2}+4 x^{2}}}\right] \\
& +\sum_{j=1}^{Z-1} \sum_{i=1}^{Z-j}\left(\sigma_{i} \alpha-1\right)\left(\sigma_{i+j} \alpha-1\right) \frac{\exp (-k j)}{j} .
\end{aligned}
$$


If we now define the Boltzmann factors

$$
x_{j}=\exp \left[\frac{-\xi \exp \left(-k \sqrt{(2 j-1-Z)^{2}+4 x^{2}}\right)}{\sqrt{(2 j-1-Z)^{2}+4 x^{2}}}\right],
$$

and

$$
y_{j}=\exp \left[\frac{-\xi \exp (-k j)}{j}\right],
$$

the contribution of each configuration to the partition function will be a product of these factors raised to exponents which are polynomials in $\alpha$, that is

$$
Q=\sum_{i=1}^{N_{c}} \prod_{j=1}^{Z / 2} x_{j}^{v_{i, j}} \prod_{j=1}^{Z-1} y_{j}^{u_{i, j}}
$$

The polynomials $v_{i, j}=-a_{i, j} \alpha+b_{i, j} \alpha^{2}$ and $u_{i, j}=c_{i, j}-d_{i, j} \alpha+e_{i, j} \alpha^{2}$, have integer non-negative coefficients. The advantage of the simple model constructed above is that for not too large values of $Z$ and $n$ the partition function can be evaluated exactly with a help of a computer.

The potential of mean force (measured in units of $q^{2} / \epsilon b$ ) between a polyion-counterion complex and an $\alpha$-ion located at $x$ is,

$$
\phi(\xi, k, \alpha, x)=-\frac{1}{\xi} \ln \frac{Q(x)}{Q(\infty)} .
$$

The potential is normalized so that $\phi(\infty)=0$.

The computer code which generates the partition function for given values of $Z$ and $n$ determines the set of integer coefficients of the polynomials defined following the Eq.(4). Each set of polynomial coefficients may correspond to more than one internal configuration of the polyion, so that the degeneracy must also be taken into account. All the data is stored on the computer and used to perform a floating point calculation of the free energy.

\section{RESULTS AND DISCUSSION}

In Fig. 2 the potential of mean force between various complexes and an $\alpha$-ion is plotted. The complexes are composed of a polyion of charge $-10 q$ and $n$ associated divalent counte-

rions. Notice that for $n=5$ (neutral complex) the potential is a monotonically increasing function of $x$, so that the sixth counterion is always attracted to the complex. 


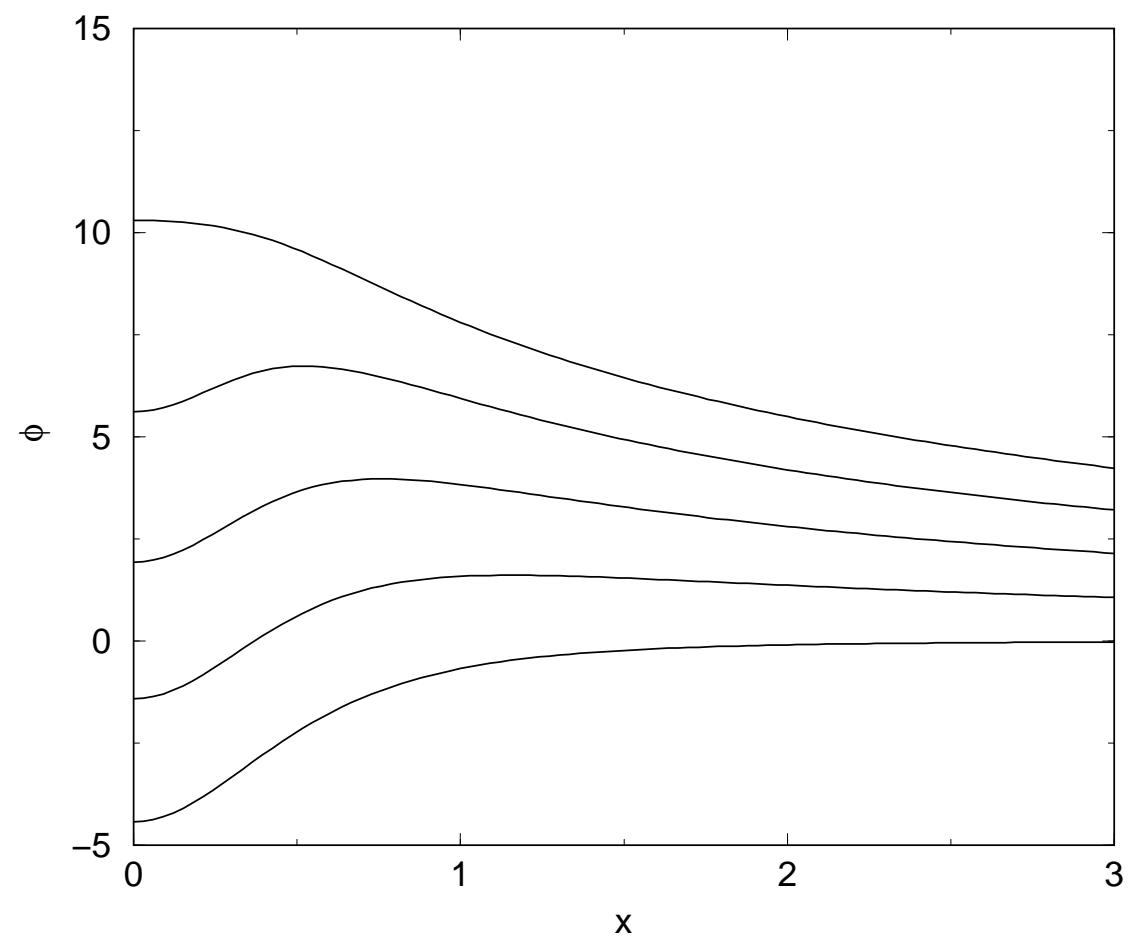

FIG. 2: Potential of mean force as a function of $x$ for $Z=10, \xi=1, k=0$, and $\alpha=2$. In upward order, the curves correspond to $n=5,6,7,8,9$ condensed counterions.

For an overcharged complex with $n=6$ condensed counterions, the potential of mean force develops a barrier. At large distances the seventh counterion is repelled from the complex, while at short distances it is attracted to it. The minimum of the free energy, however, is reached when the seventh counterion is located at $x=0$. The potential of mean force, therefore, favors counterion condensation. The size of the barrier increases with $n$ and the minimum at $x=0$ becomes metastable for $n=8$. For $n=9$ the potential is a monotonically decreasing function of $x$, and the tenth counterion is always repelled from the complex. We next study the dependence of the depth of the potential well and the height of the barrier on the parameters of the model.

In Fig. 2. we saw that when the complex is overcharged $n>Z / \alpha$, the potential can have two minima, one located at $x=\infty$ and another at $x=0$. Which one of the two minima is the global one is determined by the sign of $\phi(0)$. Figs. 3 and 4 show the behavior of $\phi(0)$ as a function of $\xi$ and $k$. When $\phi(0)<0$ the position at $x=0$ is the absolute minimum, while when $\phi(0)>0, x=0$ is at most metastable. We should note, however, that the 


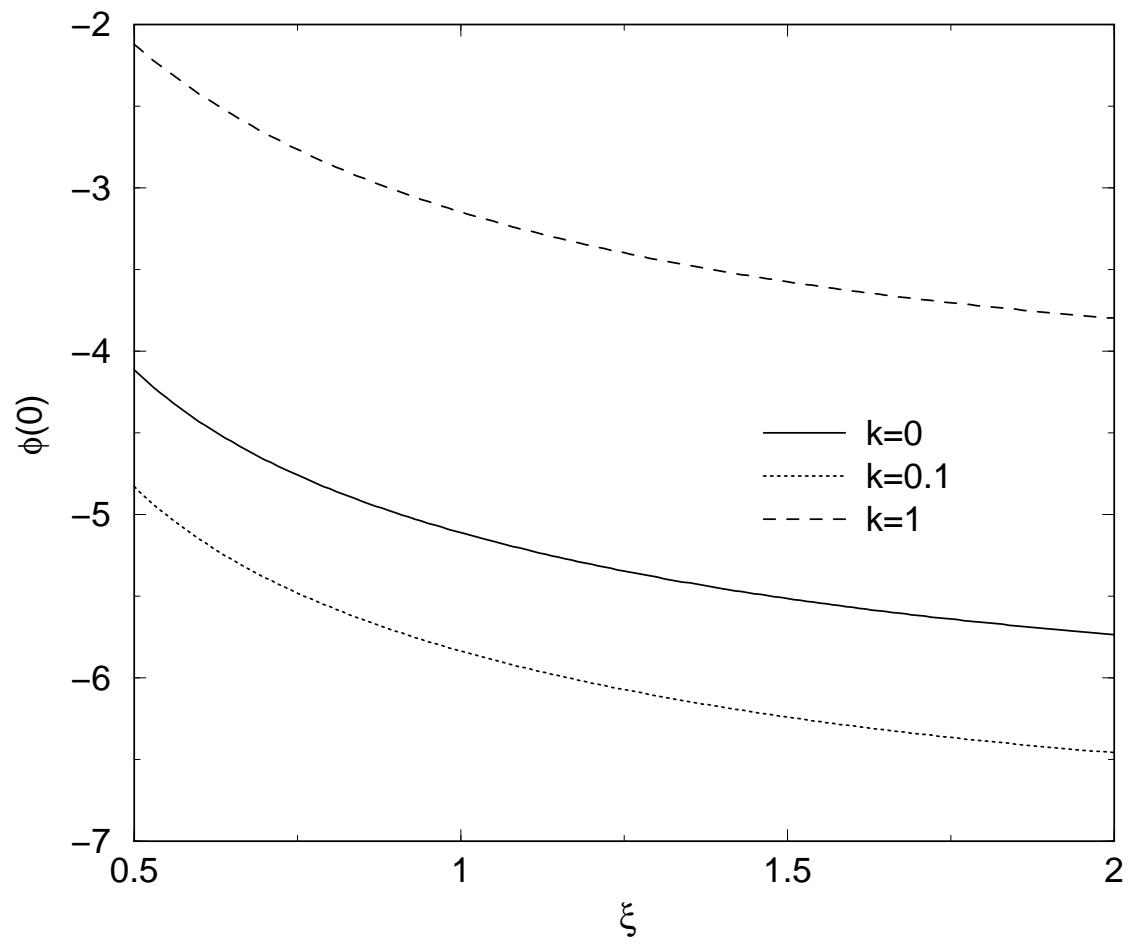

FIG. 3: The potential of mean force at $x=0$ as a function of $\xi$ for some values of $k$. The curves are for $Z=10, n=4$, and $\alpha=3$.

present discussion is not sufficient to define the absolute number of condensed counterions. For a counterion to be condensed the depth of the potential well must be sufficiently large, compared to the thermal energy $k_{B} T$, to prevent its rapid escape from the polyion surface. At the level of the present discussion this criterion is arbitrary. Thus, in this paper we will not consider the absolute number of condensed counterions but only the conditions which favor or disfavor the counterions condensation. From Figs. [3and 4 , we see that for a polyion of $Z=10$ and $n=4$ condensed trivalent counterions the minimum at $x=0$ is the global one for the parameters plotted. Approach of an additional fifth counterion to this already overcharged complex is, therefore, energetically favorable.

The depth of the global minimum $|\phi(0)|$ is a monotonically increasing function of the Manning parameter, see Fig. 3. The dependence on the salt concentration, however, is not monotonic. From Fig. 4 we see that small concentrations of salt favor counterion condensation, i.e. $\phi(0)$ becomes more negative for small $k$. Larger concentrations of salt, however, have a destabilizing effect on the counterion condensation. 


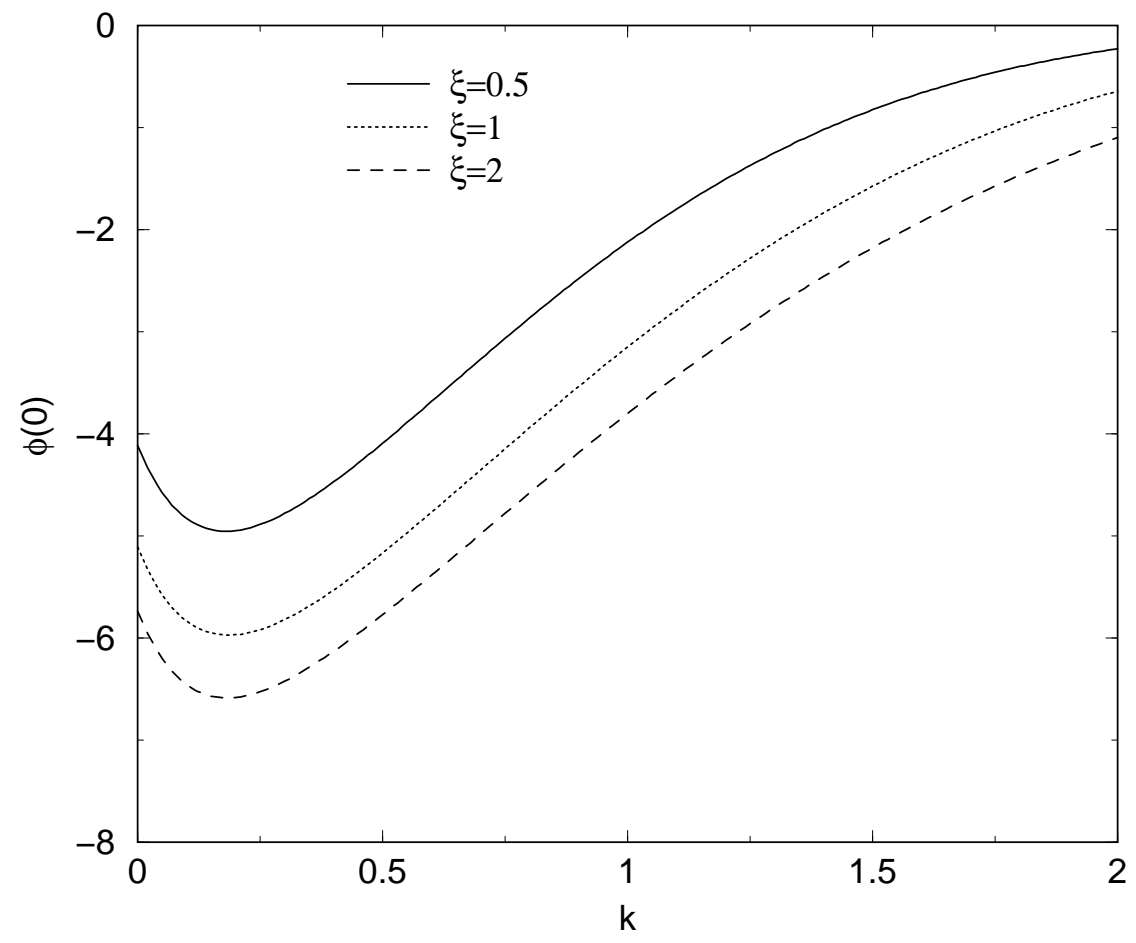

FIG. 4: $\phi(0)$ as a function of $k$ for some values of $\xi$. The curves are for $Z=10, n=4$, and $\alpha=3$.

This is even clearer for complexes composed of a polyion with $Z=10$ and $n=5$ condensed trivalent counterions. Fig. 5 shows that the position of the free energy minimum is a non-trivial function of salt concentration. Depending on the Manning parameter $\xi$ and the concentration of salt $k$, association of an additional, sixth, counterion can be either favored or disfavored. On the other hand, for $Z=10, n=6$, and $\alpha=3, \phi(0)$ is always positive so that a complex with $n=7$ condensed counterions can be at most metastable.

We next explore the dependence of the barrier height $\phi\left(x_{m}\right)$, where $x_{m}$ is the position of the maximum of the potential of mean force see Fig. 2. on the parameters of the model. In Fig. 6] $\phi\left(x_{m}\right)$ is depicted as a function of the Manning parameter $\xi$ for polyion of size $Z=10$ with $n=4$ associated counterions. We see that the barrier height diminishes with the increase of $\xi$ and the amount of salt inside the suspension.

To explore the dependence of the barrier height on the size of the polyion $Z$, in Fig. [7 we plot $\phi\left(x_{m}\right)$ as a function of $Z$ for complexes composed of a polyion and $n^{*}$ condensed trivalent 


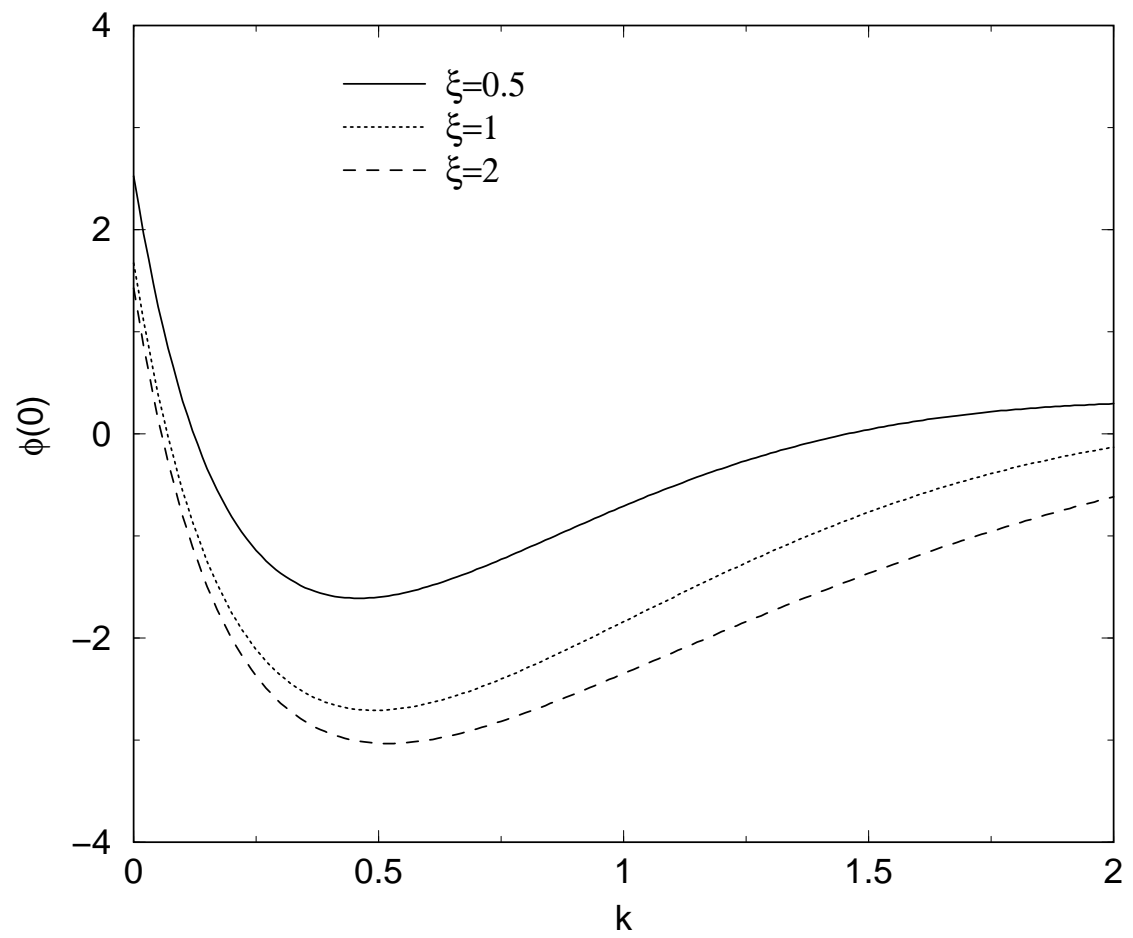

FIG. 5: $\phi(0)$ as a function of $k$ for some values of $\xi$. The curves are for $Z=10, n=5$, and $\alpha=3$.

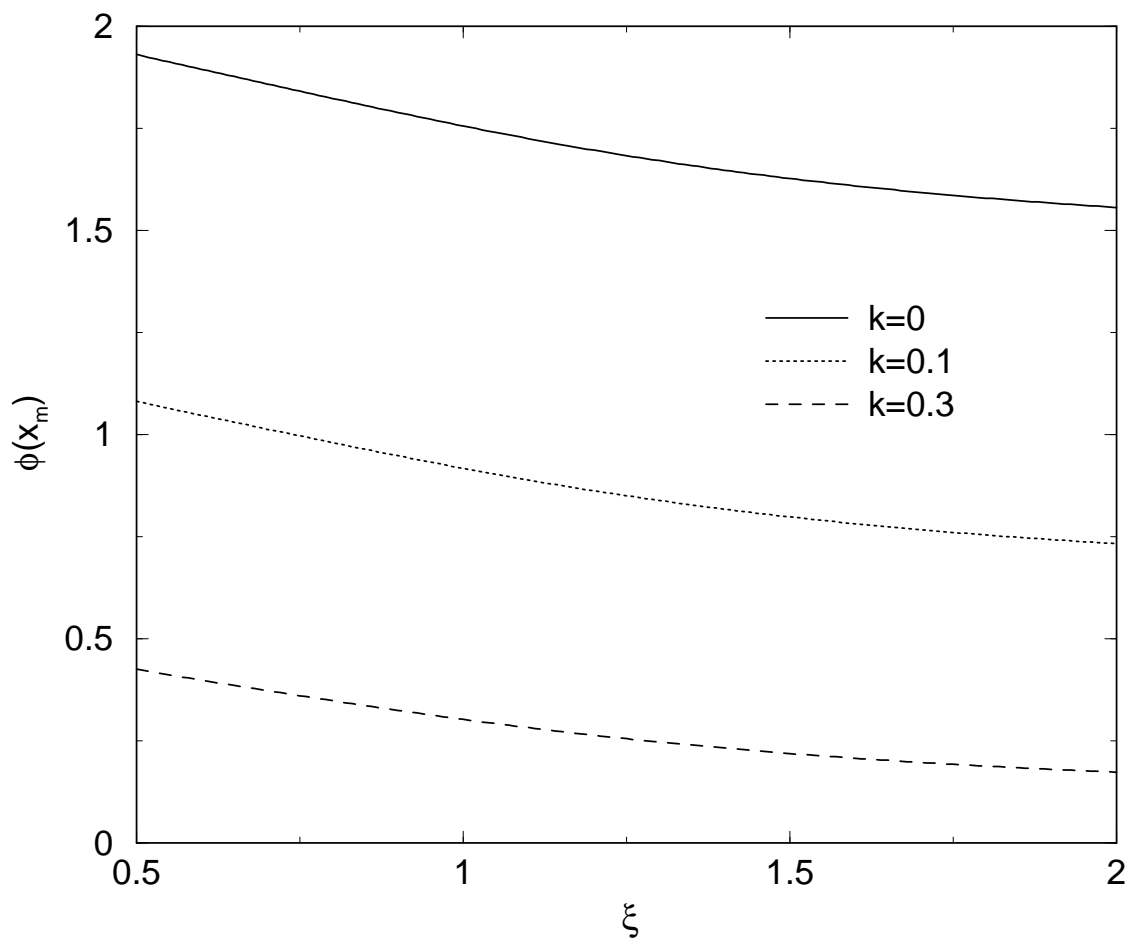

FIG. 6: The barrier height $\phi\left(x_{m}\right)$ as a function of $\xi$ for some values of $k$. The curves are for $Z=10$, $n=4$, and $\alpha=3$. 


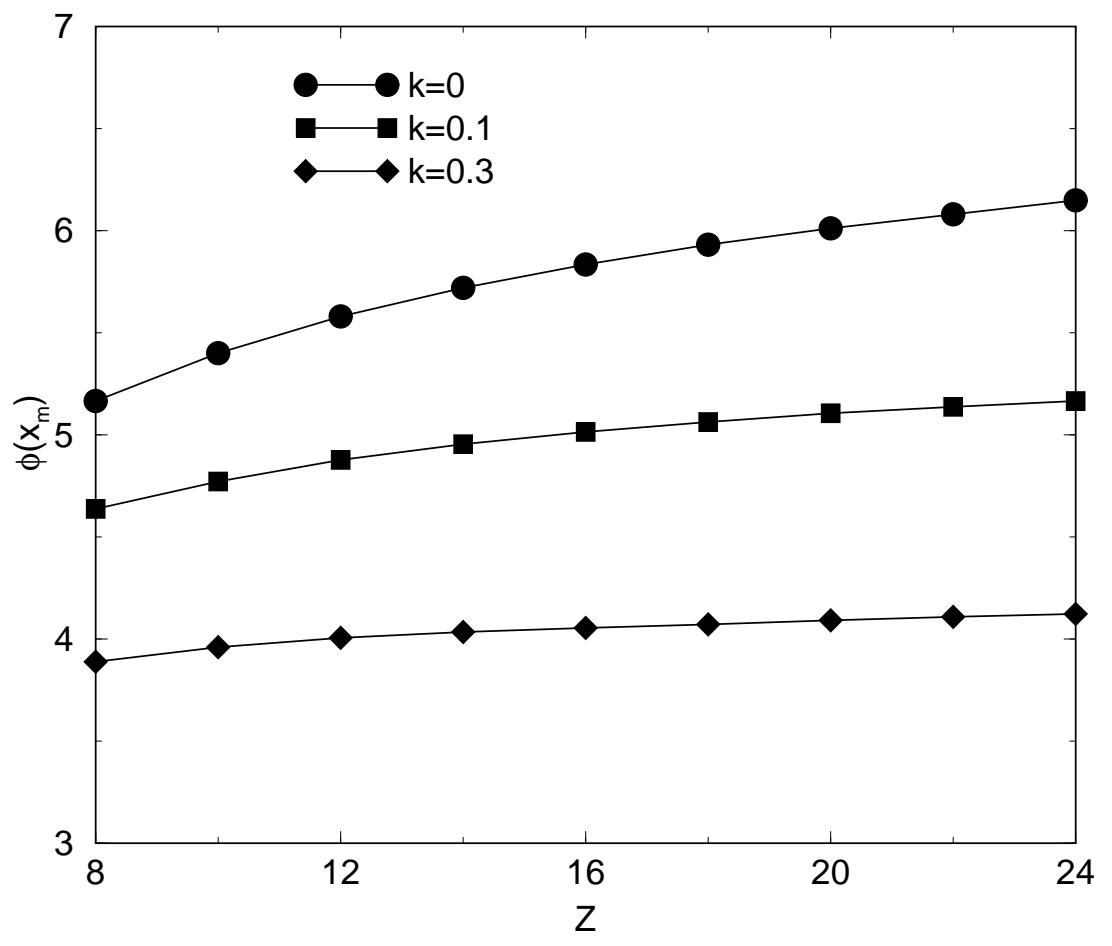

FIG. 7: The barrier for polyions with $\xi=1$ and $n^{*}$ condensed trivalent counterions, such that $\phi_{n^{*}}(0)=0$, as a function of $Z$.

counterions, such that $\phi_{n^{*}}(0)=0$. While in the absence of salt the barrier height shows a significant dependence on the polyion size, at finite salt concentration this dependence weakens and $\phi\left(x_{m}\right)$ seems to saturate when the polyion size is significantly larger than the Debye length. For large $Z$ and small concentration of electrolyte, however, the kinetic barrier can be many $k_{B} T$, providing a significant limitation to overcharging 27 .

Charge reversal is a consequence of strong positional correlations between the counterions. These correlations are induced by the electrostatic repulsion between the particles. Thus, we expect that both the barrier height and the relative depth of the absolute minimum will be strongly dependent on the counterion valence. In Figs. 8 and 9 we show the dependence of the barrier height and the depth of the potential well on the valence of the counterions. 


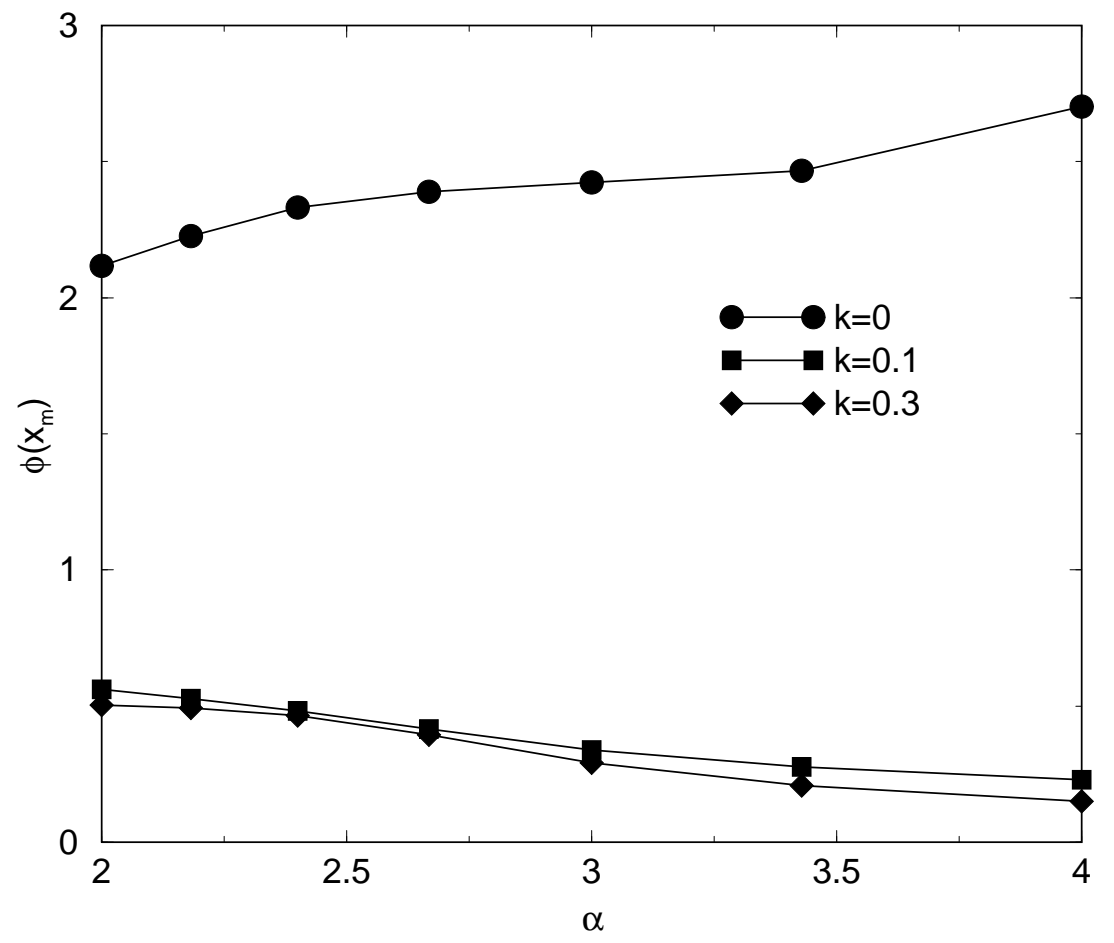

FIG. 8: The barrier for complexes composed of $Z=20$ and $n$ condensed counterions, such that $n \alpha=24$, as a function of the counterion valence $\alpha$.

Although all the overcharged complexes depicted in Figs. 8 and 9 have the same net charge $4 q$, the depth of the potential well and the height of the kinetic barrier depend on $\alpha$. As expected, larger counterion charge leads to stronger positional correlations and favors the counterion condensation and the charge reversal $(\phi(0)$ becomes more negative with increasing $\alpha)$. The barrier height, however, once again shows a nontrivial dependence on the salt concentration. For small amounts of salt and large $Z$, increased counterion valence leads to larger kinetic barriers.

\section{CONCLUSIONS}

We have studied the potential of mean force between a polyion and an $\alpha$-valent counterion inside a polyelectrolyte solution containing multivalent counterions and monovalent salt. The model is sufficiently simple that the partition function can be calculated exactly. It is found that for an overcharged polyion the potential of mean force can have two minima, one located at $x=0$ and another $x=\infty$. Which one of the minima is the global one depends on 


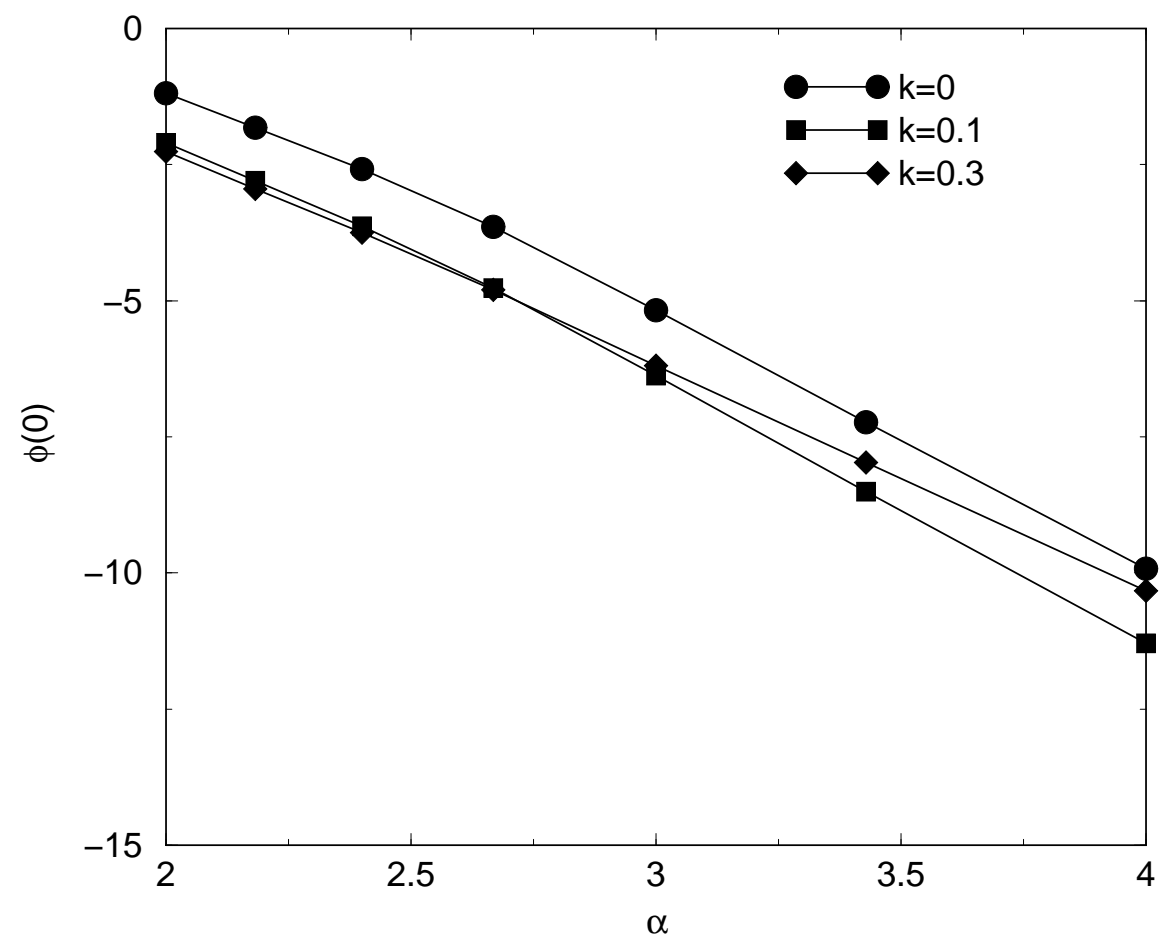

FIG. 9: $\phi(0)$ for complexes composed of $Z=20$ and $n$ condensed counterions, such that $n \alpha=24$, as a function of the counterion valence $\alpha$.

the charge density of the polyion and the amount of salt inside the suspension. When the global minimum is at $x=0$, a counterion from the bulk finds it energetically favorable to approach the polyion surface. To reach $x=0$, however, the counterion must overcome a free energy barrier. For small salt concentrations, this barrier can be sufficiently large to provide a kinetic limitation to the extent of charge reversal. Furthermore, even if the counterion reaches $x=0$, whether or not it will become condensed will depend on the depth of the potential well. Counterion condensation will occur only if $\phi\left(x_{m}\right)-\phi(0) \gg 1 / \xi$. Otherwise, the thermal fluctuations will lead to a fast escape of the counterion from the $x=0$ minimum.

For suspensions containing rodlike polyelectrolytes and the multivalent counterions micro phase separation is observed under certain conditions ${ }^{28.29}$. The polyions aggregate forming bundles with a well defined crossectional area. It has been argued that bundle formation is an activated process and the size of the bundles is kinetically controlled ${ }^{30.31 .32}$. It should then be quite interesting to explore the dependence of the barrier height on the concentration of monovalent electrolyte using a theory similar to the one presented above.

To conclude, the extent of the charge reversal is strongly dependent on the amount of 
monovalent salt present in the suspension. Small concentrations of salt will enhance the overcharging while an excessive amount of salt will hinder the charge reversal. Furthermore, even if the minimum of the free energy corresponds to an overcharged state, we find that depending on the polyion charge density and the amount of salt in the suspension, there can be significant kinetic limitations to the overcharging.

This work was supported in part by the Brazilian agencies CNPq and FAPERJ. JFS acknowledges funding by project Pronex-CNPq-FAPERJ/171.168-2003.

1 B. Widom, J. Chem. Phys. 43, 3898 (1965).

2 B. Widom, J. Chem. Phys. 81, 1030 (1984).

3 A. B. Kolomeisky and B. Widom, Faraday Discussions 112, 81 (1999).

4 Y. Levin, Rep. Prog. Phys. 65, 1577 (2002).

5 E. Luijten, M. E. Fisher, and A. Z. Panagiotopoulos, Phys. Rev. Lett. 88, 185701 (2002).

6 R. van Roij and J. P. Hansen, Phys. Rev. Lett. 79, 3082 (1997).

7 Y. Levin, M. C. Barbosa, and M. N. Tamashiro, Europhys. Lett. 41, 123 (1998).

8 A. Diehl, M. C. Barbosa, and Y. Levin, Europhys. Lett. 53, 86 (2001).

9 M. Deserno and H. H. Grünberg, Phys. Rev. E 66, 011401 (2002).

10 M. N. Tamashiro and H. Schiessel, J. Chem. Phys. 119, 1855 (2003).

11 E. Trizac and Y. Levin, Phys. Rev. E 69, 031403 (2004).

12 I. Rouzina and V. Bloomfield, J. Chem. Phys. 100, 9977 (1996).

13 N. Grønbech-Jensen, R. J. Mashl, R. F. Bruinsma, and W. M. Gelbart, Phys. Rev. Lett. 78, 2477 (1997).

14 B.-Y. Ha and A. J. Liu, Phys. Rev. Lett. 79, 1289 (1997).

15 J. J. Arenzon, J. F. Stilck, and Y. Levin, Eur. Phys. J. B 12, 79 (1999).

16 J. P. Hansen and H. Löwen, Annual Reviews of Phys. Chem, 51, 209 (2000).

17 W. M. Gelbart, R. F. Bruinsma, P. A. Pincus, and V. A. Parsegian, Physics Today 53, 38 (2000).

18 F. J. Solis and M. O. de la Cruz, Physics Today 54, 71 (2001).

19 T. E. Angelini, H. Liang, W. Wrigglers, and G. C. L. Wong, PNAS 100, 8634 (2003).

20 M. Lozada-Cassou, R. Saavedra-Barrera, and D. Henderson, J. Chem. Phys. 77, 5150 (1982). 
21 R. Messina, E. G. Tovar, M. Lozada-Cassou, and C. Holm, Europhys. Lett. 60, 383 (2002).

22 A. Y. Grosberg, T. T. Nguyen, and B. I. Shklovskii, Rev. Mod. Phys. 74, 329 (2002).

23 A. Martin-Molina, M. Quesada-Perez, F. Galisteo-Gonzalez, and R. Hidalgo-Alvarez, J. Phys.: Condensed Mat. 15, S3475 (2003).

24 P. W. Debye and E. Hückel, Phys. Z. 24, 185 (1923).

25 G. S. Manning, J. Chem. Phys. 51, 924 (1969).

26 G. S. Manning, Q. Rev. Biophys. II 2, 179 (1978).

27 Y. Levin and J. J. Arenzon, J. Phys. A: Math. Gen 36, 5857 (2003).

28 J. X. Tang and P. A. Janmey, J. Biol. Chem. 271, 8556 (1996).

29 R. Bruinsma, Phys. Rev. E 63, 061705 (2001).

30 B. I. Shklovskii, Phys.Rev.Lett. 82, 3628 (1999).

31 B.-Y. Ha and A. J. Liu, Europhys. Lett. 46, 624 (1999).

32 J. F. Stilck, Y. Levin, and J. J. Arenzon, J. Stat. Phys. 106, 287 (2002). 